\title{
Existence results for boundary value problems of arbitrary order integrodifferential equations in Banach spaces
}

\section{K. Karthikeyan and Bashir Ahmad}

\begin{abstract}
We study a boundary value problem of fractional integrodifferential equations involving Caputo's derivative of order $\alpha \in(n-1, n)$ in a Banach space. Existence and uniqueness results for the problem are established by means of the Hölder's inequality together with some standard fixed point theorems.
\end{abstract}

\section{Introduction}

The study of fractional differential equations has recently gained much attention due to extensive applications of these equations in the mathematical modelling of physical, engineering and biological phenomena. Examples and details concerning the development of the theory, methods and applications of fractional calculus can be found in the books [9], [11] and papers [1], [3], [4], [6], [7]. For some results on boundary value problems of fractional integrodifferential equations, we refer to the papers [2], [5], [12] and the references therein.

In this paper, we study the existence and uniqueness of solutions for the following boundary value problem of nonlinear fractional integrodifferential equations (BVP)

$$
\left\{\begin{array}{l}
{ }^{c} D^{\alpha} y(t)=f(t, y(t),(G y)(t),(S y)(t)), t \in J=[0, T] \\
y(0)=y_{0}, y^{\prime}(0)=y_{0}^{1}, y^{\prime \prime}(0)=y_{0}^{2}, \cdots, y^{(n-1)}(0)=y_{0}^{n-2} \\
y^{(n-1)}(T)=y_{T},
\end{array}\right.
$$

Key Words: Fractional order, integrodifferential equations, boundary conditions, existence and uniqueness, Hölder's inequality, Fixed point theorem.

2010 Mathematics Subject Classification: Primary 26A33; Secondary 34B05.

Received: March 2012.

Revised: March 2012.

Accepted: June 2012. 
where $\alpha \in(n-1, n),{ }^{c} D^{\alpha}$ is the Caputo fractional derivative of order $\alpha$, $f: J \times X \times X \rightarrow X$ is a given continuous function, $X$ is a Banach space and $y_{0}, y_{0}^{i}(i=1,2, \cdots, n-2, n>2, n$ is an integer $), y_{T}$ are some elements of $X$ and $G, S$ are integral operators given by

$$
(G y)(t)=\int_{0}^{t} k_{1}(t, s) y(s) d s,
$$

and

$$
(S y)(t)=\int_{0}^{t} k_{2}(t, s) y(s) d s
$$

with

$$
\gamma_{0}=\max \int_{0}^{t} k_{1}(t, s) d s, \gamma_{1}=\max \int_{0}^{t} k_{2}(t, s) d s,
$$

$k_{1}, k_{2} \in C\left([0, T] \times[0, T], \mathbb{R}^{+}\right)$.

The paper is organized as follows. In Section 2, we recall some concepts of fractional calculus and known results. In Section 3, we present main results: the first result is based on Banach contraction principle, while the second one is obtained by applying Schaeffer's fixed point theorem. An illustrative example is also presented.

\section{Preliminaries}

Let $C(J, X)$ denotes the Banach space of all continuous functions from $J$ into $X$ with the norm $\|y\|_{\infty}:=\sup \{\|y(t)\|: t \in J\}$. For measurable functions $m: J \rightarrow \mathbb{R}$, define the norm $\|m\|_{L^{p}(J, \mathbb{R})}=\left(\int_{J}|m(t)|^{p} d t\right)^{\frac{1}{p}}<\infty, 1 \leq p<\infty$, where $L^{p}(J, \mathbb{R})$ is the Banach space of all Lebesgue measurable functions.

Now let us recall some basic concepts of fractional calculus ([8], [10]).

Definition 2.1. For at least $(n-1)$-times continuously differentiable function $h:[0, \infty) \rightarrow \mathbb{R}$, the Caputo derivative of fractional order $q$ is defined as

$$
{ }^{c} D^{q} h(t)=\frac{1}{\Gamma(n-q)} \int_{0}^{t}(t-s)^{n-q-1} h^{(n)}(s) d s, \quad n-1<q<n, n=[q]+1,
$$

where $[q]$ denotes the integer part of the real number $q$. 
Definition 2.2. The Riemann-Liouville fractional integral of order $q$ is defined as

$$
I^{q} h(t)=\frac{1}{\Gamma(q)} \int_{0}^{t} \frac{h(s)}{(t-s)^{1-q}} d s, \quad q>0,
$$

provided the integral exists.

Lemma 2.1[8]. For $\alpha>0$, the general solution of fractional differential equation ${ }^{c} D^{\alpha} h(t)=0$ is

$$
h(t)=c_{0}+c_{1} t+c_{2} t^{2}+\cdots+c_{n-1} t^{n-1},
$$

where $c_{i} \in \mathbb{R}, i=0,1,2, \cdots, n-1$.

Lemma 2.2. Let $\alpha>0$. Then

$$
I^{\alpha}\left({ }^{c} D^{\alpha} h\right)(t)=h(t)+c_{0}+c_{1} t+c_{2} t^{2}+\cdots+c_{n-1} t^{n-1},
$$

for some $c_{i} \in \mathbb{R}, i=0,1,2, \cdots, n-1, n=-[-\alpha]$.

Definition 2.3. A function $y \in C(J, X)$ with its $\alpha$-derivative existing on $J$ is said to be a solution of the fractional BVP (1.1) if $y$ satisfies the equation ${ }^{c} D^{\alpha} y(t)=f(t, y(t),(G y)(t),(S y)(t))$ a.e. on $J$, and the conditions $y(0)=$ $y_{0}, y^{\prime}(0)=y_{0}^{1}, y^{\prime \prime}(0)=y_{0}^{2}, \cdots, y^{(n-1)}(0)=y_{0}^{n-2}, y^{(n-1)}(T)=y_{T}$.

To define the solution for problem (1), we need the following auxiliary lemma.

Lemma 2.3. Let $\bar{f}: J \rightarrow X$ be a continuous. A function $y \in C(J, X)$ is a solution of the fractional integral equation

$$
\begin{aligned}
y(t)= & \frac{1}{\Gamma(\alpha)} \int_{0}^{t}(t-s)^{\alpha-1} \bar{f}(s) d s \\
& -\frac{t^{n-1}}{(n-1) ! \Gamma(\alpha-n+1)} \int_{0}^{T}(T-s)^{\alpha-n} \bar{f}(s) d s \\
& +y_{0}+y_{0}^{1} t+\frac{y_{0}^{2}}{2 !} t^{2}+\cdots+\frac{y_{0}^{n-2}}{(n-2) !} t^{n-2}+\frac{y_{T}}{(n-1) !} t^{n-1},
\end{aligned}
$$

if and only if $y$ is a solution of the problem

$$
\left\{\begin{array}{l}
{ }^{c} D^{\alpha} y(t)=\bar{f}(t), t \in J=[0, T], \alpha \in(n-1, n), \\
y(0)=y_{0}, y^{\prime}(0)=y_{0}^{1}, y^{\prime \prime}(0)=y_{0}^{2}, \cdots, y^{(n-1)}(0)=y_{0}^{n-2} \\
y^{(n-1)}(T)=y_{T}
\end{array}\right.
$$


Proof. The proof is based on Lemma 2.2 and employs the standard arguments, for instance, see [1]. So we omit it.

In view of Lemma 2.3, we define the solution of (1) as follows.

Lemma 2.4. Let $f: J \times X \times X \times X \rightarrow X$ be continuous function. Then $y \in C(J, X)$ is a solution of the fractional integral equation

$$
\begin{aligned}
y(t)= & \frac{1}{\Gamma(\alpha)} \int_{0}^{t}(t-s)^{\alpha-1} f(s, y(s),(G y)(s),(S y(s))) d s \\
& -\frac{t^{n-1}}{(n-1) ! \Gamma(\alpha-n+1)} \int_{0}^{T}(T-s)^{\alpha-n} f(s, y(s),(G y)(s),(S y(s))) d s \\
& +y_{0}+y_{0}^{1} t+\frac{y_{0}^{2}}{2 !} t^{2}+\cdots+\frac{y_{0}^{n-2}}{(n-2) !} t^{n-2}+\frac{y_{T}}{(n-1) !} t^{n-1},
\end{aligned}
$$

if and only if $y$ is a solution of the fractional BVP (1.1)

In the sequel, we need the following results.

Lemma 2.5. (Bochner theorem) A measurable function $f: J \rightarrow X$ is Bochner integrable if $\|f\|$ is Lebesgue integrable.

Lemma 2.6. (Mazur lemma) If $\mathcal{K}$ is a compact subset of $X$, then its convex closure $\overline{c o n v} \mathcal{K}$ is compact.

Lemma 2.7. (Ascoli-Arzela theorem) Let $\mathcal{S}=\{s(t)\}$ is a function family of continuous mappings $s:[a, b] \rightarrow X$. If $\mathcal{S}$ is uniformly bounded and equicontinuous, and for any $t^{*} \in[a, b]$, the set $\left\{s\left(t^{*}\right)\right\}$ is relatively compact, then there exists a uniformly convergent function sequence $\left\{s_{n}(t)\right\}(n=1,2, \cdots, t \in[a, b])$ in $\mathcal{S}$.

Theorem 2.8. (Schaeffer's fixed point theorem). Let $F: X \rightarrow X$ be completely continuous operator. If the set

$$
E(F)=\left\{x \in X: x=\lambda^{*} F x \text { for some } \lambda^{*} \in[0,1]\right\}
$$

is bounded, then $F$ has fixed points.

\section{Main results}

To prove the main results, we introduce the following assumptions: 
$\left(H_{1}\right)$ The function $f: J \times X \times X \times X \rightarrow X$ is strongly measurable with respect to $t$ on $J$.

$\left(H_{2}\right)$ There exists a constant $\alpha_{1} \in(0, \alpha)$ and real-valued functions $m_{1}(t)$, $m_{2}(t), m_{3}(t) \in L^{\frac{1}{\alpha_{1}}}(J, X)$ such that

$$
\begin{aligned}
& \|f(t, x(t),(G x)(t),(S x)(t))-f(t, y(t),(G y)(t),(S y)(t))\| \\
\leq & m_{1}(t)\|x-y\|+m_{2}(t)\|G x-G y\|+m_{3}(t)\|S x-S y\|,
\end{aligned}
$$

for each $t \in J$ and $x, y \in X$.

$\left(H_{3}\right)$ There exists a constant $\alpha_{2} \in(0, \alpha)$ and real-valued function $h(t) \in$ $L^{\frac{1}{\alpha_{2}}}(J, X)$ such that

$$
\| f(t, y,(G y),(S y) \| \leq h(t), \text { for each } t \in J \text { and all } y \in X,
$$

with $H=\|h\|_{L^{\frac{1}{\alpha_{2}}}(J, X)}$.

$\left(H_{4}\right)$ The function $f: J \times X \times X \times X \rightarrow X$ is continuous.

$\left(H_{5}\right)$ There exist constants $\lambda \in\left[0,1-\frac{1}{p}\right)$ for some $1<p<\frac{1}{1-\alpha}$ and $N>0$ such that

$\|f(t, u, G u, S u)\| \leq N\left(1+\gamma_{0}\|u\|^{\lambda}+\gamma_{1}\|u\|^{\lambda}\right)$ for each $t \in J$ and all $u \in X$.

Our first result is based on Banach's contraction principle. For the sake of convenience, we set the notation:

$$
M=\left\|m_{1}+\gamma_{0} m_{2}+\gamma_{1} m_{3}\right\|_{L^{\frac{1}{\alpha_{1}}}(J, X)} .
$$

Theorem 3.1. Assume that $\left(H_{1}\right)-\left(H_{3}\right)$ hold. If

$\Omega_{\alpha, T, n}=\frac{M}{\Gamma(\alpha)} \frac{T^{\alpha-\alpha_{1}}}{\left(\frac{\alpha-\alpha_{1}}{1-\alpha_{1}}\right)^{1-\alpha_{1}}}+\frac{M}{(n-1) ! \Gamma(\alpha-n+1)} \frac{T^{\alpha-\alpha_{1}}}{\left(\frac{\alpha-\alpha_{1}-n+1}{1-\alpha_{1}}\right)^{1-\alpha_{1}}}<1$,

then the problem (1) has a unique solution on $J$.

Proof. For each $t \in J$, we have

$$
\begin{aligned}
& \int_{0}^{t}\left\|(t-s)^{\alpha-1} f(s, y(s),(G y)(s),(S y(s)))\right\| d s \\
\leq & \left(\int_{0}^{t}(t-s)^{\frac{\alpha-1}{1-\alpha_{2}}} d s\right)^{1-\alpha_{2}}\left(\int_{0}^{t}(h(s))^{\frac{1}{\alpha_{2}}} d s\right)^{\alpha_{2}}
\end{aligned}
$$




$$
\begin{aligned}
& \leq\left(\int_{0}^{t}(t-s)^{\frac{\alpha-1}{1-\alpha_{2}}} d s\right)^{1-\alpha_{2}}\left(\int_{0}^{T}(h(s))^{\frac{1}{\alpha_{2}}} d s\right)^{\alpha_{2}} \\
& \leq \frac{T^{\alpha-\alpha_{2}} H}{\left(\frac{\alpha-\alpha_{2}}{1-\alpha_{2}}\right)^{1-\alpha_{2}}} .
\end{aligned}
$$

Thus, $\left\|(t-s)^{\alpha-1} f(s, y(s),(S y)(s),(G y(s)))\right\|$ is Lebesgue integrable with respect to $s \in[0, t]$ for all $t \in J$ and $y \in C(J, X)$. Then $(t-s)^{\alpha-1} f(s, y(s)$, $(G y)(s),(S y(s)))$ is Bochner integrable with respect to $s \in[0, t]$ for all $t \in J$ by Lemma 2.5. Since

$$
\begin{aligned}
& \int_{0}^{T}\left\|(T-s)^{\alpha-n} f(s, y(s),(G y)(s),(S y(s)))\right\| d s \\
\leq & \left(\int_{0}^{T}(T-s)^{\frac{\alpha-n}{1-\alpha_{2}}} d s\right)^{1-\alpha_{2}}\left(\int_{0}^{T}(h(s))^{\frac{1}{\alpha_{2}}} d s\right)^{\alpha_{2}} \\
\leq & \frac{T^{\alpha-\alpha_{2}-n+1} H}{\left(\frac{\alpha-\alpha_{2}-n+1}{1-\alpha_{2}}\right)^{1-\alpha_{2}}},
\end{aligned}
$$

therefore, $\left\|(T-s)^{\alpha-n} f(s, y(s),(G y)(s),(S y)(s))\right\|$ is Lebesgue integrable with respect to $s \in[0, T]$ for all $t \in J$ and $y \in C(J, X)$. Hence $(T-s)^{\alpha-n} f(s, y(s)$, $(G y)(s),(S y)(s))$ is Bochner integrable with respect to $s \in[0, T]$ for all $t \in J$ by Lemma 2.5 .

Let us choose

$$
\begin{aligned}
r \geq & \frac{H T^{\alpha-\alpha_{2}}}{\Gamma(\alpha)\left(\frac{\alpha-\alpha_{2}}{1-\alpha_{2}}\right)^{1-\alpha_{2}}}+\frac{H T^{\alpha-\alpha_{2}}}{(n-1) ! \Gamma(\alpha-n+1)}\left(\frac{1-\alpha_{2}}{\alpha-\alpha_{2}-n+1}\right)^{1-\alpha_{2}} \\
& +\left\|y_{0}\right\|+\left\|y_{0}^{1}\right\| T+\frac{\left\|y_{0}^{2}\right\|}{2 !} T^{2}+\cdots+\frac{\left\|y_{0}^{n-2}\right\|}{(n-2) !} T^{n-2}+\frac{\left\|y_{T}\right\|}{(n-1) !} T^{n-1} .
\end{aligned}
$$

Now we define an operator $F$ on $B_{r}:=\{y \in C(J, X):\|y\| \leq r\}$ by

$$
\begin{aligned}
(F y)(t) & =\frac{1}{\Gamma(\alpha)} \int_{0}^{t}(t-s)^{\alpha-1} f(s, y(s),(G y)(s),(S y)(s)) d s \\
& -\frac{t^{n-1}}{(n-1) ! \Gamma(\alpha-n+1)} \int_{0}^{T}(T-s)^{\alpha-n} f(s, y(s),(G y)(s),(S y)(s)) d s \\
& +y_{0}+y_{0}^{1} t+\frac{y_{0}^{2}}{2 !} t^{2}+\cdots+\frac{y_{0}^{n-2}}{(n-2) !} t^{n-2}+\frac{y_{T}}{(n-1) !} t^{n-1}, \quad t \in J
\end{aligned}
$$

Observe that the problem (1) has solutions if the operator $F$ has fixed points on $B_{r}$. It will be shown by means of Banach contraction principle that $F$ has a fixed point. The proof is divided into two steps. 
Step 1. Fy $\in B_{r}$ for every $y \in B_{r}$.

For every $y \in B_{r}$ and any $\delta>0$, by $\left(H_{3}\right)$ and the Hölder's inequality, we get

$$
\begin{aligned}
& \|(F y)(t+\delta)-(F y)(t)\| \\
& \leq \| \frac{1}{\Gamma(\alpha)} \int_{0}^{t+\delta}(t+\delta-s)^{\alpha-1} f(s, y(s),(G y)(s),(S y)(s)) d s \\
& -\frac{1}{\Gamma(\alpha)} \int_{0}^{t}(t-s)^{\alpha-1} f(s, y(s),(G y)(s),(S y)(s)) d s \| \\
& +\| \frac{(t+\delta)^{n-1}}{(n-1) ! \Gamma(\alpha-n+1)} \int_{0}^{T}(T-s)^{\alpha-n} f(s, y(s),(G y)(s),(S y)(s)) d s \\
& -\frac{t^{n-1}}{(n-1) ! \Gamma(\alpha-n+1)} \int_{0}^{T}(T-s)^{\alpha-n} f(s, y(s),(G y)(s),(S y)(s)) d s \| \\
& +\| y_{0}^{1}(t+\delta-t)+\frac{y_{0}^{2}}{2 !}\left[(t+\delta)^{2}-t^{2}\right]+\cdots+\frac{y_{0}^{n-2}}{(n-2) !}\left[(t+\delta)^{n-2}-t^{n-2}\right] \\
& +\frac{y_{T}}{(n-1) !}\left[(t+\delta)^{n-1}-t^{n-1}\right] \| \\
& \leq \frac{1}{\Gamma(\alpha)} \int_{0}^{t}\left[(t+\delta-s)^{\alpha-1}-(t-s)^{\alpha-1}\right]\|f(s, y(s),(G y)(s),(S y)(s))\| d s \\
& +\frac{1}{\Gamma(\alpha)} \int_{t}^{t+\delta}(t+\delta-s)^{\alpha-1}\|f(s, y(s),(G y)(s),(S y)(s))\| d s \\
& +\frac{\left[(t+\delta)^{n-1}-t^{n-1}\right]}{(n-1) ! \Gamma(\alpha-n+1)} \int_{0}^{T}(T-s)^{\alpha-n}\|f(s, y(s),(G y)(s),(S y)(s))\| d s \\
& +\left\|y_{0}^{1}\right\|(t+\delta-t)+\frac{\left\|y_{0}^{2}\right\|}{2 !}\left[(t+\delta)^{2}-t^{2}\right]+\cdots+\frac{\left\|y_{0}^{n-2}\right\|}{(n-2) !}\left[(t+\delta)^{n-2}-t^{n-2}\right] \\
& +\frac{\left\|y_{T}\right\|}{(n-1) !}\left[(t+\delta)^{n-1}-t^{n-1}\right] \\
& \leq \frac{1}{\Gamma(\alpha)} \int_{0}^{t}\left[(t+\delta-s)^{\alpha-1}-(t-s)^{\alpha-1}\right] h(s) d s \\
& +\frac{1}{\Gamma(\alpha)} \int_{t}^{t+\delta}(t+\delta-s)^{\alpha-1} h(s) d s \\
& +\frac{\left[(t+\delta)^{n-1}-t^{n-1}\right]}{(n-1) ! \Gamma(\alpha-n+1)} \int_{0}^{T}(T-s)^{\alpha-n} h(s) d s \\
& +\left\|y_{0}^{1}\right\|(t+\delta-t)+\frac{\left\|y_{0}^{2}\right\|}{2 !}\left[(t+\delta)^{2}-t^{2}\right]+\cdots+\frac{\left\|y_{0}^{n-2}\right\|}{(n-2) !}\left[(t+\delta)^{n-2}-t^{n-2}\right] \\
& +\frac{\left\|y_{T}\right\|}{(n-1) !}\left[(t+\delta)^{n-1}-t^{n-1}\right]
\end{aligned}
$$




$$
\begin{aligned}
& \leq \frac{1}{\Gamma(\alpha)}\left(\int_{0}^{t}\left[(t+\delta-s)^{\alpha-1}-(t-s)^{\alpha-1}\right]^{\frac{1}{1-\alpha_{2}}} d s\right)^{1-\alpha_{2}}\left(\int_{0}^{t}(h(s))^{\frac{1}{\alpha_{2}}} d s\right)^{\alpha_{2}} \\
& +\frac{1}{\Gamma(\alpha)}\left(\int_{t}^{t+\delta}(t+\delta-s)^{\frac{\alpha-1}{1-\alpha_{2}}} d s\right)^{1-\alpha_{2}}\left(\int_{t}^{t+\delta}(h(s))^{\frac{1}{\alpha_{2}}} d s\right)^{\alpha_{2}} \\
& +\frac{\left[(t+\delta)^{n-1}-t^{n-1}\right]}{(n-1) ! \Gamma(\alpha-n+1)}\left(\int_{0}^{T}(T-s)^{\frac{\alpha-n}{1-\alpha_{2}}} d s\right)^{1-\alpha_{2}}\left(\int_{0}^{T}(h(s))^{\frac{1}{\alpha_{2}}} d s\right)^{\alpha_{2}} \\
& +\left\|y_{0}^{1}\right\|(t+\delta-t)+\frac{\left\|y_{0}^{2}\right\|}{2 !}\left[(t+\delta)^{2}-t^{2}\right]+\cdots+\frac{\left\|y_{0}^{n-2}\right\|}{(n-2) !}\left[(t+\delta)^{n-2}-t^{n-2}\right] \\
& +\frac{\left\|y_{T}\right\|}{(n-1) !}\left[(t+\delta)^{n-1}-t^{n-1}\right] \\
& \leq \frac{1}{\Gamma(\alpha)}\left(\int_{0}^{t}\left[(t+\delta-s)^{\frac{\alpha-1}{1-\alpha_{2}}}-(t-s)^{\frac{\alpha-1}{1-\alpha_{2}}}\right] d s\right)^{1-\alpha_{2}}\left(\int_{0}^{t}(h(s))^{\frac{1}{\alpha_{2}}} d s\right)^{\alpha_{2}} \\
& +\frac{1}{\Gamma(\alpha)}\left(\int_{t}^{t+\delta}(t+\delta-s)^{\frac{\alpha-1}{1-\alpha_{2}}} d s\right)^{1-\alpha_{2}}\left(\int_{t}^{t+\delta}(h(s))^{\frac{1}{\alpha_{2}}} d s\right)^{\alpha_{2}} \\
& +\frac{\left[(t+\delta)^{n-1}-t^{n-1}\right]}{(n-1) ! \Gamma(\alpha-n+1)}\left(\int_{0}^{T}(T-s)^{\frac{\alpha-n}{1-\alpha_{2}}} d s\right)^{1-\alpha_{2}}\left(\int_{0}^{T}(h(s))^{\frac{1}{\alpha_{2}}} d s\right)^{\alpha_{2}} \\
& +\left\|y_{0}^{1}\right\|(t+\delta-t)+\frac{\left\|y_{0}^{2}\right\|}{2 !}\left[(t+\delta)^{2}-t^{2}\right]+\cdots+\frac{\left\|y_{0}^{n-2}\right\|}{(n-2) !}\left[(t+\delta)^{n-2}-t^{n-2}\right] \\
& +\frac{\left\|y_{T}\right\|}{(n-1) !}\left[(t+\delta)^{n-1}-t^{n-1}\right] \\
& \leq \frac{H}{\Gamma(\alpha)}\left(\frac{(t+\delta)^{\frac{\alpha-\alpha_{2}}{1-\alpha_{2}}}}{\frac{\alpha-\alpha_{2}}{1-\alpha_{2}}}-\frac{\delta^{\frac{\alpha-\alpha_{2}}{1-\alpha_{2}}}}{\frac{\alpha-\alpha_{2}}{1-\alpha_{2}}}-\frac{t^{\frac{\alpha-\alpha_{2}}{1-\alpha_{2}}}}{\frac{\alpha-\alpha_{2}}{1-\alpha_{2}}}\right)^{1-\alpha_{2}} \\
& +\frac{H}{\Gamma(\alpha)}\left(\frac{\delta^{\frac{\alpha-\alpha_{2}}{1-\alpha_{2}}}}{\frac{\alpha-\alpha_{2}}{1-\alpha_{2}}}\right)^{1-\alpha_{2}}+\frac{\left[(t+\delta)^{n-1}-t^{n-1}\right]}{(n-1) ! \Gamma(\alpha-n+1)} \frac{T^{\alpha-\alpha_{2}-n+1} H}{\left(\frac{\alpha-\alpha_{2}-n+1}{1-\alpha_{2}}\right)^{1-\alpha_{2}}} \\
& +\left\|y_{0}^{1}\right\|(t+\delta-t)+\frac{\left\|y_{0}^{2}\right\|}{2 !}\left[(t+\delta)^{2}-t^{2}\right]+\cdots+\frac{\left\|y_{0}^{n-2}\right\|}{(n-2) !}\left[(t+\delta)^{n-2}-t^{n-2}\right] \\
& +\frac{\left\|y_{T}\right\|}{(n-1) !}\left[(t+\delta)^{n-1}-t^{n-1}\right] .
\end{aligned}
$$

It is obvious that the right-hand side of the above inequality tends to zero as $\delta \rightarrow 0$. Therefore, $F$ is continuous on $J$, that is, $F y \in C(J, X)$. Moreover, for $y \in B_{r}$ and all $t \in J$, we get

$$
\begin{aligned}
& \|(F y)(t)\| \\
\leq & \frac{1}{\Gamma(\alpha)} \int_{0}^{t}(t-s)^{\alpha-1}\|f(s, y(s),(G y)(s),(S y)(s))\| d s
\end{aligned}
$$




$$
\begin{aligned}
& +\frac{t^{n-1}}{(n-1) ! \Gamma(\alpha-n+1)} \int_{0}^{T}(T-s)^{\alpha-n}\|f(s, y(s),(G y)(s),(S y)(s))\| d s \\
& +\left\|y_{0}\right\|+\left\|y_{0}^{1}\right\| T+\frac{\left\|y_{0}^{2}\right\|}{2 !} T^{2}+\cdots+\frac{\left\|y_{0}^{n-2}\right\|}{(n-2) !} T^{n-2}+\frac{\left\|y_{T}\right\|}{(n-1) !} T^{n-1} \\
& \leq \frac{1}{\Gamma(\alpha)} \int_{0}^{t}(t-s)^{\alpha-1} h(s) d s \\
& -\frac{t^{n-1}}{(n-1) ! \Gamma(\alpha-n+1)} \int_{0}^{T}(T-s)^{\alpha-n} h(s) d s \\
& +\left\|y_{0}\right\|+\left\|y_{0}^{1}\right\| T+\frac{\left\|y_{0}^{2}\right\|}{2 !} T^{2}+\cdots+\frac{\left\|y_{0}^{n-2}\right\|}{(n-2) !} T^{n-2}+\frac{\left\|y_{T}\right\|}{(n-1) !} T^{n-1} \\
& \leq \frac{1}{\Gamma(\alpha)}\left(\int_{0}^{t}(t-s)^{\frac{\alpha-1}{1-\alpha_{2}}} d s\right)^{1-\alpha_{2}}\left(\int_{0}^{t}(h(s))^{\frac{1}{\alpha_{2}}} d s\right)^{\alpha_{2}} \\
& +\frac{\left[(t+\delta)^{n-1}-(t)^{n-1}\right]}{(n-1) ! \Gamma(\alpha-n+1)}\left(\int_{0}^{T}(T-s)^{\frac{\alpha-n}{1-\alpha_{2}}} d s\right)^{1-\alpha_{2}}\left(\int_{0}^{T}(h(s))^{\frac{1}{\alpha_{2}}} d s\right)^{\alpha_{2}} \\
& +\left\|y_{0}\right\|+\left\|y_{0}^{1}\right\| T+\frac{\left\|y_{0}^{2}\right\|}{2 !} T^{2}+\cdots+\frac{\left\|y_{0}^{n-2}\right\|}{(n-2) !} T^{n-2}+\frac{\left\|y_{T}\right\|}{(n-1) !} T^{n-1} \\
& \leq \frac{H T^{\alpha-\alpha_{2}}}{\Gamma(\alpha)\left(\frac{\alpha-\alpha_{2}}{1-\alpha_{2}}\right)^{1-\alpha_{2}}}+\frac{H T^{\alpha-\alpha_{2}}}{(n-1) ! \Gamma(\alpha-n+1)\left(\frac{\alpha-\alpha_{2}-n+1}{1-\alpha_{2}}\right)^{1-\alpha_{2}}} \\
& +\left\|y_{0}\right\|+\left\|y_{0}^{1}\right\| T+\frac{\left\|y_{0}^{2}\right\|}{2 !} T^{2}+\cdots+\frac{\left\|y_{0}^{n-2}\right\|}{(n-2) !} T^{n-2}+\frac{\left\|y_{T}\right\|}{(n-1) !} T^{n-1} \\
& \leq r,
\end{aligned}
$$

which implies that $\|F y\|_{\infty} \leq r$. Thus, we can conclude that for all $y \in$ $B_{r}, F y \in B_{r}$, that is, $F: B_{r} \rightarrow B_{r}$.

Step 2. $F$ is a contraction mapping on $B_{r}$.

For $x, y \in B_{r}$ and any $t \in J$, using (H2), the Hölder's inequality and

$$
\begin{aligned}
& \|f(s, x(s),(G x)(s),(S x)(s))-f(s, y(s),(G y)(s),(S y)(s))\| \\
\leq & m_{1}(s)\|x(s)-y(s)\|+m_{2}(s)\|G x(s)-G y(s)\|+m_{3}(s)\|S x(s)-S y(s)\| \\
= & \rho(s),
\end{aligned}
$$

we get

$$
\begin{aligned}
& \|(F x)(t)-(F y)(t)\| \\
\leq & \frac{1}{\Gamma(\alpha)} \int_{0}^{t}(t-s)^{\alpha-1} \rho(s) d s+\frac{t^{n-1}}{(n-1) ! \Gamma(\alpha-n+1)} \int_{0}^{T}(T-s)^{\alpha-n} \rho(s) d s \\
\leq & \frac{\|x-y\|_{\infty}}{\Gamma(\alpha)}\left(\int_{0}^{t}(t-s)^{\frac{\alpha-1}{1-\alpha_{1}}} d s\right)^{1-\alpha_{1}}
\end{aligned}
$$




$$
\begin{aligned}
& \times\left(\int_{0}^{t}\left(m_{1}(s)+\gamma_{0} m_{2}(s)+\gamma_{1} m_{3}(s)\right)^{\frac{1}{\alpha_{1}}} d s\right)^{\alpha_{1}} \\
& +\frac{T^{n-1}}{(n-1) ! \Gamma(\alpha-n+1)}\left(\int_{0}^{T}(T-s)^{\frac{\alpha-n}{1-\alpha_{1}}} d s\right)^{1-\alpha_{1}} \\
& \times\left(\int_{0}^{T}\left(m_{1}(s)+\gamma_{0} m_{2}(s)+\gamma_{1} m_{3}(s)\right)^{\frac{1}{\alpha_{1}}} d s\right)^{\alpha_{1}} \\
\leq & \left(\frac{M}{\Gamma(\alpha)} \frac{T^{\alpha-\alpha_{1}}}{\Gamma(\alpha)\left(\frac{\alpha-\alpha_{1}}{1-\alpha_{1}}\right)^{1-\alpha_{1}}}+\frac{M}{(n-1) ! \Gamma(\alpha-n+1)} \frac{T^{\alpha-\alpha_{1}}}{\left(\frac{\alpha-\alpha_{1}-n+1}{1-\alpha_{1}}\right)^{1-\alpha_{1}}}\right) \\
& \times\|x-y\|_{\infty} .
\end{aligned}
$$

So we obtain

$$
\|F x-F y\|_{\infty} \leq \Omega_{\alpha, T, n}\|x-y\|_{\infty} .
$$

Thus, $F$ is contraction by the condition (3). Hence, by Banach contraction mapping principle, the operator $F$ has a unique fixed point which is the unique solution of the problem (1).

Our next result is based on Schaeffer's fixed point theorem.

Theorem 3.2. Assume that $\left(H_{1}\right),\left(H_{4}\right)$ and $\left(H_{5}\right)$ hold. Then the problem (1) has at least one solution on $J$.

Proof. As before, let $F: C(J, X) \rightarrow C(J, X)$ be the operator defined by (4). We will show that $F$ satisfies the hypotheses of Theorem 2.8. The proof consists of several steps.

Step 1. $F$ is a continuous operator.

Let $\left\{y_{n}\right\}$ be a sequence such that $y_{n} \rightarrow y$ in $C(J, X)$. Then for each $t \in J$, using the continuity of $f$, we have

$$
\begin{aligned}
& \left\|F y_{n}-F y\right\|_{\infty} \\
\leq & \left(\frac{T^{\alpha}}{\Gamma(\alpha+1)}+\frac{T^{\alpha}}{(n-1) ! \Gamma(\alpha-n+2)}\right) \\
& \times\left\|f\left(\cdot, y_{n}(\cdot),(G y)_{n}(\cdot),(S y)_{n}(\cdot)\right)-f(\cdot, y(\cdot),(G y)(\cdot),(S y)(\cdot))\right\|_{\infty} \rightarrow 0 \\
& \text { as } n \rightarrow \infty .
\end{aligned}
$$

Step 2. $F$ maps bounded sets into bounded sets in $C(J, X)$.

Indeed, it is enough to show that for any $\eta^{*}>0$, there exists a $l>0$ such that for each $y \in B_{\eta^{*}}=\left\{y \in C(J, X):\|y\|_{\infty} \leq \eta^{*}\right\}$, we have $\|F y\|_{\infty} \leq l$.

For each $t \in J$, by $\left(H_{4}\right)$, we get

$$
\|(F y)(t)\|
$$




$$
\begin{aligned}
\leq & \frac{N\left(1+\gamma_{0}\|y\|^{\lambda}+\gamma_{1}\|y\|^{\lambda}\right)}{\Gamma(\alpha)} \int_{0}^{t}(t-s)^{\alpha-1} d s \\
& +\frac{N\left(1+\gamma_{0}\|y\|^{\lambda}+\gamma_{1}\|y\|^{\lambda}\right) T^{n-1}}{(n-1) ! \Gamma(\alpha-n+1)} \int_{0}^{T}(T-s)^{\alpha-n} d s \\
& +\left\|y_{0}\right\|+\left\|y_{0}^{1}\right\| T+\frac{\left\|y_{0}^{2}\right\|}{2 !} T^{2}+\cdots+\frac{\left\|y_{0}^{n-2}\right\|}{(n-2) !} T^{n-2}+\frac{\left\|y_{T}\right\|}{(n-1) !} T^{n-1} \\
\leq & \frac{N\left(1+\gamma_{0}\left(\eta^{*}\right)^{\lambda}+\gamma_{1}\left(\eta^{*}\right)^{\lambda}\right)}{\Gamma(\alpha)} \int_{0}^{t}(t-s)^{\alpha-1} d s \\
& +\frac{T^{n-1} N\left(\left(1+\gamma_{0}\left(\eta^{*}\right)^{\lambda}+\gamma_{1}\left(\eta^{*}\right)^{\lambda}\right)\right.}{(n-1) ! \Gamma(\alpha-n+1)} \int_{0}^{T}(T-s)^{\alpha-n} d s \\
& +\left\|y_{0}\right\|+\left\|y_{0}^{1}\right\| T+\frac{\left\|y_{0}^{2}\right\|}{2 !} T^{2}+\cdots+\frac{\left\|y_{0}^{n-2}\right\|}{(n-2) !} T^{n-2}+\frac{\left\|y_{T}\right\|}{(n-1) !} T^{n-1} \\
\leq & \left(\frac{1}{\Gamma(\alpha+1)}+\frac{1}{(n-1) ! \Gamma(\alpha-n+2)}\right)^{\alpha} N\left(1+\gamma_{0}\left(\eta^{*}\right)^{\lambda}+\gamma_{1}\left(\eta^{*}\right)^{\lambda}\right) \\
& +\left\|y_{0}\right\|+\left\|y_{0}^{1}\right\| T+\frac{\left\|y_{0}^{2}\right\|}{2 !} T^{2}+\cdots+\frac{\left\|y_{0}^{n-2}\right\|}{(n-2) !} T^{n-2}+\frac{\left\|y_{T}\right\|}{(n-1) !} T^{n-1}:=l,
\end{aligned}
$$

which implies that

$$
\|F y\|_{\infty} \leq l
$$

Step 3. $F$ maps bounded sets into equicontinuous sets of $C(J, X)$.

Let $0 \leq t_{1}<t_{2} \leq T, y \in B_{\eta^{*}}$. Using $\left(H_{4}\right)$ again, we have

$$
\begin{aligned}
& \left\|(F y)\left(t_{2}\right)-(F y)\left(t_{1}\right)\right\| \\
\leq & \frac{N\left(1+\gamma_{0}\|y\|^{\lambda}+\gamma_{1}\|y\|^{\lambda}\right)}{\Gamma(\alpha)} \int_{0}^{t_{1}}\left[\left(t_{2}-s\right)^{\alpha-1}-\left(t_{1}-s\right)^{\alpha-1}\right] d s \\
& +\frac{N\left(1+\gamma_{0}\|y\|^{\lambda}+\gamma_{1}\|y\|^{\lambda}\right)}{\Gamma(\alpha)} \int_{t_{1}}^{t_{2}}\left(t_{2}-s\right)^{\alpha-1} d s \\
& +\frac{t_{2}^{n-1}-t_{1}^{n-1}}{(n-1) ! \Gamma(\alpha-n+1)} \int_{0}^{T}(T-s)^{\alpha-n} N\left(1+\gamma_{0}\|y\|^{\lambda}+\gamma_{1}\|y\|^{\lambda}\right) d s \\
& +\left\|y_{0}^{1}\right\|\left(t_{2}-t_{1}\right)+\frac{\left\|y_{0}^{2}\right\|}{2 !}\left(t_{2}^{2}-t_{1}^{2}\right)+\cdots+\frac{\left\|y_{0}^{n-2}\right\|}{(n-2) !}\left(t_{2}^{n-2}-t_{1}^{n-2}\right) \\
& +\frac{\left\|y_{T}\right\|}{(n-1) !}\left(t_{2}^{n-1}-t_{1}^{n-1}\right) \\
\leq & \frac{N\left(1+\gamma_{0}\left(\eta^{*}\right)^{\lambda}+\gamma_{1}\left(\eta^{*}\right)^{\lambda}\right)}{\Gamma(\alpha)}\left(\int_{0}^{t_{1}}\left[\left(t_{2}-s\right)^{\alpha-1}-\left(t_{1}-s\right)^{\alpha-1}\right] d s\right.
\end{aligned}
$$




$$
\begin{aligned}
& \left.+\int_{t_{1}}^{t_{2}}\left(t_{2}-s\right)^{\alpha-1} d s\right) \\
& +\frac{\left(t_{2}^{2}-t_{1}^{2}\right) N\left(\left(1+\gamma_{0}\left(\eta^{*}\right)^{\lambda}+\gamma_{1}\left(\eta^{*}\right)^{\lambda}\right)\right.}{(n-1) ! \Gamma(\alpha-n+1)} \int_{0}^{T}(T-s)^{\alpha-n} d s \\
& +\left\|y_{0}^{1}\right\|\left(t_{2}-t_{1}\right)+\frac{\left\|y_{0}^{2}\right\|}{2 !}\left(t_{2}^{2}-t_{1}^{2}\right)+\cdots+\frac{\left\|y_{0}^{n-2}\right\|}{(n-2) !}\left(t_{2}^{n-2}-t_{1}^{n-2}\right) \\
& +\frac{\left\|y_{T}\right\|}{(n-1) !}\left(t_{2}^{n-1}-t_{1}^{n-1}\right) \\
& \leq+\frac{N\left(1+\gamma_{0}\left(\eta^{*}\right)^{\lambda}+\gamma_{1}\left(\eta^{*}\right)^{\lambda}\right)}{\Gamma(\alpha+1)}\left(t_{2}^{\alpha}-t_{1}^{\alpha}\right) \\
& +\frac{T^{\alpha-n+1} N\left(1+\gamma_{0}\left(\eta^{*}\right)^{\lambda}+\gamma_{1}\left(\eta^{*}\right)^{\lambda}\right)}{(n-1) ! \Gamma(\alpha-n+2)}\left(t_{2}^{n-1}-t_{1}^{n-1}\right) \\
& +\left\|y_{0}^{1}\right\|\left(t_{2}-t_{1}\right)+\frac{\left\|y_{0}^{2}\right\|}{2 !}\left(t_{2}^{2}-t_{1}^{2}\right)+\cdots+\frac{\left\|y_{0}^{n-2}\right\|}{(n-2) !}\left(t_{2}^{n-2}-t_{1}^{n-2}\right) \\
& +\frac{\left\|y_{T}\right\|}{(n-1) !}\left(t_{2}^{n-1}-t_{1}^{n-1}\right) .
\end{aligned}
$$

As $t_{2} \rightarrow t_{1}$, the right-hand side of the above inequality tends to zero, therefore $F$ is equicontinuous.

Now, let $\left\{y_{n}\right\}, n=1,2, \cdots$ be a sequence on $B_{\eta^{*}}$, and

$$
\left(F y_{n}\right)(t)=\left(F_{1} y_{n}\right)(t)+\left(F_{2} y_{n}\right)(t)+\left(F_{3} y\right)(t), \quad t \in J,
$$

where

$$
\begin{aligned}
\left(F_{1} y_{n}\right)(t)= & \frac{1}{\Gamma(\alpha)} \int_{0}^{t}(t-s)^{\alpha-1} f\left(s, y_{n}(s),(G y)_{n}(s),(S y)_{n}(s)\right) d s, \quad t \in J, \\
\left(F_{2} y_{n}\right)(t)= & -\frac{t^{n-1}}{(n-1) ! \Gamma(\alpha-n+1)} \\
& \times \int_{0}^{T}(T-s)^{\alpha-n} f\left(s, y_{n}(s),(G y)_{n}(s),(S y)_{n}(s)\right) d s, \quad t \in J, \\
\left(F_{3} y\right)(t)= & y_{0}+y_{0}^{1} t+\frac{y_{0}^{2}}{2 !} t^{2}+\cdots+\frac{y_{0}^{n-2}}{(n-2) !} t^{n-2}+\frac{y_{T}}{(n-1) !} t^{n-1}, \quad t \in J .
\end{aligned}
$$

In view of the condition $(H 5)$ and Lemma 2.6, we know that $\overline{c o n v} K_{1}$ is compact. For any $t^{*} \in J$,

$$
\left(F_{1} y_{n}\right)\left(t^{*}\right)=\frac{1}{\Gamma(\alpha)} \lim _{k \rightarrow \infty} \sum_{i=1}^{k} \frac{t^{*}}{k}\left(t^{*}-\frac{i t^{*}}{k}\right)^{\alpha-1}
$$




$$
\begin{aligned}
& \left.\times f\left(\frac{i t^{*}}{k}, y_{n}\left(\frac{i t^{*}}{k}\right),\left(G y_{n}\right)\left(\frac{i t^{*}}{k}\right),\left(S y_{n}\right)\left(\frac{i t^{*}}{k}\right)\right)\right) \\
& =\frac{t^{*}}{\Gamma(\alpha)} \xi_{n 1},
\end{aligned}
$$

where

$$
\left.\xi_{n 1}=\lim _{k \rightarrow \infty} \sum_{i=1}^{k} \frac{1}{k}\left(t^{*}-\frac{i t^{*}}{k}\right)^{\alpha-1} f\left(\frac{i t^{*}}{k}, y_{n}\left(\frac{i t^{*}}{k}\right),\left(G y_{n}\right)\left(\frac{i t^{*}}{k}\right),\left(S y_{n}\right)\left(\frac{i t^{*}}{k}\right)\right)\right) .
$$

Since $\overline{c o n v} K_{1}$ is convex and compact, we know that $\xi_{n 1} \in \overline{\text { conv }} K_{1}$. Hence, for any $t^{*} \in J$, the set $\left\{\left(F_{1} y_{n}\right)\left(t^{*}\right)\right\}$ is relatively compact. By Lemma 2.7 , every sequence $\left\{\left(F_{1} y_{n}\right)(t)\right\}$ contains a uniformly convergent subsequence $\left\{\left(F_{1} y_{n_{k}}\right)(t)\right\}$, $k=1,2, \cdots$ on $J$. Thus, the set $\left\{F_{1} y: y \in B_{\eta^{*}}\right\}$ is relatively compact.

\section{Set}

$$
\begin{aligned}
\left(\overline{F_{2}} y_{n}\right)(t)= & -\frac{t^{n-1}}{(n-1) ! \Gamma(\alpha-n+1)} \\
& \times \int_{0}^{t}(t-s)^{\alpha-n} f\left(s, y_{n}(s),\left(G y_{n}\right)(s),\left(S y_{n}\right)(s)\right) d s, \quad t \in J,
\end{aligned}
$$

For any $t^{*} \in J$,

$$
\begin{aligned}
& \left(\overline{F_{3}} y_{n}\right)\left(t^{*}\right)=-\frac{\left(t^{*}\right)^{n-1}}{(n-1) ! \Gamma(\alpha-n+1)} \\
& \times \lim _{k \rightarrow \infty} \sum_{i=1}^{k} \frac{t^{*}}{k}\left(t^{*}-\frac{i t^{*}}{k}\right)^{\alpha-n} f\left(\frac{i t^{*}}{k}, y_{n}\left(\frac{i t^{*}}{k}\right),\left(G y_{n}\right)\left(\frac{i t^{*}}{k}\right),\left(S y_{n}\right)\left(\frac{i t^{*}}{k}\right)\right) \\
& =-\frac{\left(t^{*}\right)^{n}}{(n-1) ! \Gamma(\alpha-n+1)} \xi_{n 2},
\end{aligned}
$$

where

$\xi_{n 2}=\lim _{k \rightarrow \infty} \sum_{i=1}^{k} \frac{1}{k}\left(t^{*}-\frac{i t^{*}}{k}\right)^{\alpha-n} f\left(\frac{i t^{*}}{k}, y_{n}\left(\frac{i t^{*}}{k}\right),\left(G y_{n}\right)\left(\frac{i t^{*}}{k}\right),\left(S y_{n}\right)\left(\frac{i t^{*}}{k}\right)\right)$.

Since $\overline{c o n v} K_{2}$ is convex and compact, we know that $\xi_{n 2} \in \overline{c o n v} K_{2}$. Hence, for any $t^{*} \in J$, the set $\left\{\left(\overline{F_{2}} y_{n}\right)\left(t^{*}\right)\right\}$ is relatively compact. By Lemma 2.7, every $\left\{\left(\overline{F_{2}} y_{n}\right)(t)\right\}$ contains a uniformly convergent subsequence $\left\{\left(\overline{F_{2}} y_{n_{k}}\right)(t)\right\}, k=$ $1,2, \cdots$ on $J$. In particular, the sequence $\left\{\left(F_{2} y_{n}\right)(t)\right\}$ contains a uniformly convergent subsequence $\left\{\left(F_{2} y_{n_{k}}\right)(t)\right\}, k=1,2, \cdots$ on $J$. Thus, the set $\left\{F_{2} y\right.$ : 
$\left.y \in B_{\eta^{*}}\right\}$ is relatively compact.

Similarly, the set $\left\{F_{3} y: y \in B_{\eta^{*}}\right\}$ is relatively compact. As a result, the set $\left\{F y: y \in B_{\eta^{*}}\right\}$ is relatively compact. In view of steps $1-3$, we conclude that $F$ is continuous and completely continuous.

Step 4. A priori bounds.

Now it remains to show that the set

$$
E(F)=\left\{y \in C(J, X): y=\lambda^{*} F y, \text { for some } \lambda^{*} \in[0,1]\right\}
$$

is bounded.

Let $y \in E(F)$, then $y=\lambda^{*} F y$ for some $\lambda^{*} \in[0,1]$. Thus, for each $t \in J$, we have

$$
\begin{aligned}
y(t) & =\lambda^{*}\left(\frac{1}{\Gamma(\alpha)} \int_{0}^{t}(t-s)^{\alpha-1} f\left(s, y_{n}(s),\left(G y_{n}\right)(s),\left(S y_{n}\right)(s)\right) d s\right. \\
- & \frac{t^{n-1}}{(n-1) ! \Gamma(\alpha-n+1)} \int_{0}^{T}(T-s)^{\alpha-n} f\left(s, y_{n}(s),\left(G y_{n}\right)(s),\left(S y_{n}\right)(s)\right) d s \\
+ & \left.y_{0}+y_{0}^{1} t+\frac{y_{0}^{2}}{2 !} t^{2}+\cdots+\frac{y_{0}^{n-2}}{(n-2) !} t^{n-2}+\frac{y_{T}}{(n-1) !} t^{n-1}\right) .
\end{aligned}
$$

For each $t \in J$, we have

$$
\begin{aligned}
\|y(t)\| \leq & \|(F y)(t)\| \\
\leq & \left.\frac{N\left(1+\gamma_{0}\|y\|^{\lambda}+\gamma_{1}\|y\|^{\lambda}\right)}{\Gamma(\alpha)} \int_{0}^{t}(t-s)^{\alpha-1}\right) d s \\
& +\frac{N\left(1+\gamma_{0}\|y\|^{\lambda}+\gamma_{1}\|y\|^{\lambda}\right) T^{n-1}}{(n-1) ! \Gamma(\alpha-n+1)} \int_{0}^{T}(T-s)^{\alpha-n} d s \\
& +\left\|y_{0}\right\|+\left\|y_{0}^{1}\right\| T+\frac{\left\|y_{0}^{2}\right\|}{2 !} T^{2}+\cdots+\frac{\left\|y_{0}^{n-2}\right\|}{(n-2) !} T^{n-2}+\frac{\left\|y_{T}\right\|}{(n-1) !} T^{n-1} \\
\leq & N\left(1+\gamma_{0}\|y\|^{\lambda}+\gamma_{1}\|y\|^{\lambda}\right) T^{\alpha}\left(\frac{1}{\Gamma(\alpha+1)}+\frac{1}{(n-1) ! \Gamma(\alpha-n+1)}\right) \\
& +\left\|y_{0}\right\|+\left\|y_{0}^{1}\right\| T+\frac{\left\|y_{0}^{2}\right\|}{2 !} T^{2}+\cdots+\frac{\left\|y_{0}^{n-2}\right\|}{(n-2) !} T^{n-2}+\frac{\left\|y_{T}\right\|}{(n-1) !} T^{n-1} \\
& =M^{*} \text { (say). }
\end{aligned}
$$

Thus, for every $t \in J$, we have

$$
\|y\|_{\infty} \leq M^{*} .
$$

This shows that the set $E(F)$ is bounded. Hence, Theorem 2.8 applies and $F$ has a fixed point which is a solution of the problem (1). 
Example 3.1. Let us define $X=\left\{y=\left(y_{1}, y_{2}, \ldots, y_{p}, \ldots\right): y_{p} \rightarrow 0\right\}$ with the norm $\|y\|=\sup _{p}\left|y_{p}\right|$ and consider the following fractional boundary value problem

$$
\left\{\begin{aligned}
&{ }^{c} D^{15 / 4} y_{p}(t)=\frac{t\left|y_{p}\right|}{3 p\left(1+\left|y_{p}\right|\right)}+\frac{1}{2 p} \int_{0}^{t} e^{-(s-t)} y_{p}(s) d s \\
&+\frac{1}{p} \int_{0}^{t} e^{-(s-t) / 2} y_{p}(s) d s \\
& y_{p}(0)=0, \quad y_{p}^{\prime}(0)=0, \quad y_{p}^{\prime \prime}(0)=0, y_{p}(1)=0 .
\end{aligned}\right.
$$

where $t \in[0,1], f=\left(f_{1}, f_{2}, \ldots, f_{p}, \ldots\right)$ with

$$
\begin{gathered}
f_{p}=\frac{t\left|y_{p}\right|}{3 p\left(1+\left|y_{p}\right|\right)}+\frac{1}{2 p} \int_{0}^{t} e^{-(s-t)} y_{p}(s) d s+\frac{1}{p} \int_{0}^{t} e^{-(s-t) / 2} y_{p}(s) d s, \\
G_{p} y(t)=\frac{1}{2 p} \int_{0}^{t} e^{-(s-t)} y_{p}(s) d s, S_{p} y(t)=\frac{1}{p} \int_{0}^{t} e^{-(s-t) / 2} y_{p}(s) d s, \\
k_{1}(t, s)=e^{-(s-t)}, k_{2}(t, s)=e^{-(s-t) / 2}, \alpha_{1}=(0,15 / 4) . \text { Moreover, } \\
\left\|f\left(t, x_{p}(t),\left(G x_{p}\right)(t),\left(S x_{p}\right)(t)\right)-f\left(t, y_{p}(t),\left(G y_{p}\right)(t),\left(S y_{p}\right)(t)\right)\right\| \\
\leq \quad m_{1}(t)\left\|x_{p}-y_{p}\right\|+m_{2}(t)\left\|G x_{p}-G y_{p}\right\|+m_{3}(t)\left\|S x_{p}-S y_{p}\right\|, \\
m_{1}(t)=t / 3, m_{2}(t)=1 / 2, m_{1}(t)=1, \gamma_{0}=\max \int_{0}^{t} k_{1}(t, s) d s=e-1, \gamma_{1}= \\
\max \int_{0}^{t} k_{2}(t, s) d s=2(\sqrt{e}-1) . \text { Clearly } \\
\|f(t, x(t),(G x)(t),(S x)(t))-f(t, y(t),(G y)(t),(S y)(t))\| \\
\leq m_{1}(t)\|x-y\|+m_{2}(t)\|G x-G y\|+m_{3}(t)\|S x-S y\| .
\end{gathered}
$$

Furthermore, with $\alpha_{1}=1 / 2, \alpha=15 / 4, n=4, T=1$, we have

$$
\begin{aligned}
M & =\left\|m_{1}+\gamma_{0} m_{2}+\gamma_{1} m_{3}\right\|_{L^{\frac{1}{\alpha_{1}}}([0,1], X)} \simeq 2.325242, \\
\Omega_{\alpha, T, n} & =\frac{M}{\Gamma(\alpha)} \frac{T^{\alpha-\alpha_{1}}}{\left(\frac{\alpha-\alpha_{1}}{1-\alpha_{1}}\right)^{1-\alpha_{1}}}+\frac{M}{(n-1) ! \Gamma(\alpha-n+1)} \frac{T^{\alpha-\alpha_{1}}}{\left(\frac{\alpha-\alpha_{1}-n+1}{1-\alpha_{1}}\right)^{1-\alpha_{1}}} \\
\simeq & 0.653451<1,
\end{aligned}
$$

Thus, all the assumptions of Theorem 3.1 are satisfied. Therefore, the conclusion of Theorem 3.1 applies to the problem (5). 


\section{Acknowledgment}

The authors are grateful to the reviewer for a careful reading of the manuscript.

\section{References}

[1] R.P. Agarwal, M. Benchohra, S. Hamani, A survey on existence results for boundary value problems of nonlinear fractional differential equations and inclusions. Acta Appl. Math. 109 (2010), 9731033.

[2] B. Ahmad, J.J. Nieto, Existence results for nonlinear boundary value problems of fractional integrodifferential equations with integral boundary conditions, Bound. Value Probl. 2009, Art. ID 708576, 11 pp.

[3] B. Ahmad, Existence of solutions for irregular boundary value problems of nonlinear fractional differential equations, Appl. Math. Lett. 23 (2010), 390-394.

[4] B. Ahmad, S.K. Ntouyas, A four-point nonlocal integral boundary value problem for fractional differential equations of arbitrary order, Electron. J. Qual. Theory Differ. Equ. 2011, No. 22, 15pp.

[5] B. Ahmad, On nonlocal boundary value problems for nonlinear integrodifferential equations of arbitrary fractional order, Results in Mathematics, DOI: $10.1007 / \mathrm{s} 00025-011-0187-9$, in press.

[6] A. Anguraj, P. Karthikeyan, and G. M. N'Guérékata, Nonlocal Cauchy problem for some fractional abstract integrodifferential equations in $\mathrm{Ba}-$ nach space, Commun. Appl. Anal. 55 (2009), 1-6.

[7] D. Baleanu, O.G. Mustafa, R.P. Agarwal, An existence result for a superlinear fractional differential equation, Appl. Math. Lett. 23 (2010), $1129-1132$.

[8] A.A. Kilbas, H.M. Srivastava, J.J. Trujillo, Theory and Applications of Fractional Differential Equations, North-Holland Mathematics Studies, 204. Elsevier Science B.V., Amsterdam, 2006.

[9] V. Lakshmikantham, S. Leela, J.V. Devi, Theory of Fractional Dynamic Systems, Cambridge Scientific Publishers, Cambridge (2009)

[10] I. Podlubny, Fractional Differential Equations, Academic Press, San Diego, 1999. 
[11] J. Sabatier, O.P. Agrawal, J.A.T. Machado (Eds.), Advances in Fractional Calculus: Theoretical Developments and Applications in Physics and Engineering, Springer, Dordrecht, 2007.

[12] J.R. Wang, Y. Zhou, W. Wei, H. Xu, Nonlocal problems for fractional integrodifferential equations via fractional operators and optimal controls, Comput. Math. Appl. 62 (2011), 1427-1441.

K. Karthikeyan

Department of Mathematics, KSR College of Technology

Tiruchengode-637 215, Tamilnadu, India.

Email: karthi_phd2010@yahoo.co.in

Bashir Ahmad

Department of Mathematics, Faculty of Science, King Abdulaziz University

P. O. Box 80203, Jeddah 21589, Saudi Arabia

Email: bashirahmad_qau@yahoo.com 